\title{
Intuitionistic fuzzy negations and their use in image classification
}

\author{
Alžbeta Michalíková ${ }^{1,2}$ \\ ${ }^{1}$ Faculty of Natural Sciences, Matej Bel University \\ Tajovského 40, Banská Bystrica, Slovakia \\ e-mail: alzbeta.michalikova@umb.sk \\ ${ }^{2}$ Mathematical Institute, Slovak Academy of Sciences \\ Ďumbierska 1, Banská Bystrica, Slovakia
}

Received: 11 June 2020

Accepted: 27 September 2020

\begin{abstract}
In this paper, the problem of classification of images is discussed. Our specific problem is that we need to classify tire images into selected classes. The classes are characterized by some patterns. In the first step images are represented as the vectors. Then the membership and non-membership value to each coordinate of the vector is calculated and the theory of intuitionistic fuzzy sets is used. In [7] the classification of images was performed with respect to the valued of so called Sim function, which was defined as a ratio of distance between pattern data and image data and distance between pattern data and the complement of image data. The complement of image data was obtained by using specific intuitionistic fuzzy negation. In [2] a list of 53 intuitionistic fuzzy negations was presented. We have decided to use some of these negations to improve the results of classification.
\end{abstract}

Keywords: Intuitionistic fuzzy sets, Intuitionistic fuzzy negations, Similarity measure, Image classification.

2010 Mathematics Subject Classification: 03C98, 03E72.

\section{Introduction}

The research presented in this paper is motivated by several consultations with active crime scene investigators. During these consultations, it became apparent, that criminology department is in 
critical need of advanced software for tire tread print identification. The current software used by crime scene investigators is outdated, time demanding and hard to work with. This led to development of new software tool for tire print identification. Prototype of this software is still being developed by department of Computer Science of Matej Bel University. The problem can be formalized as follows: While on the crime scene, criminologists often find various types of prints, such as foot prints, finger prints and one of most common types of prints - tire tread prints. If there is a possibility to recognize the brand or manufacturer of tires present on the crime scene, they could be one of the important evidence which could contribute to the conviction of offender. To recognize the tire tread brand or manufacturer, the database of tire tread prints needs to be build (see $[3,5])$. The images of the tire treads could be obtained from the web pages. Not all images are in the required quality and in the proper position, but the access to these images is simple. In our previous work, presented in [9], we described process of creating database of downloaded tire tread images. We developed the web crawling application to extract the relevant images together with important information from the predefined web pages. Not all images are suitable for the additional processing and for comparing with the tire tread prints obtained at the crime scene. In [10] authors presented seven basic types/classes of the tire tread images and they described the advantages/disadvantages of each class from the point of comparison mentioned in the paper [10].

This paper presents one of the first steps of tire tread print identification - tire tread position identification - by using functions defined on intuitionistic fuzzy sets. The images obtained from the web crawling application [9] are classified into the seven classes. The classes of tire tread images used in this article are displayed on Figure 1.

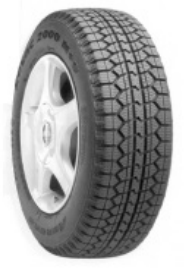

(a) Class 1

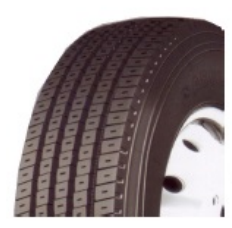

(b) Class 2

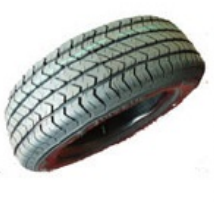

(c) Class 3

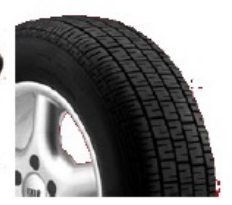

(d) Class 4

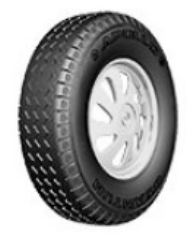

(e) Class 5

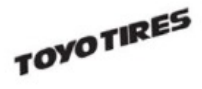

(f) Class 6



(g) Class 7

Figure 1. Used classes of tire tread images

The next step of processing of the images is the specification of the tire tread prints position. The position of tire tread print is specific for each class. As an example, the position of tire tread print of the Class 1 is presented on the Figure 2b. After some pre-procesing one could get the tire treat print as shown on Figure 2c. This type of image could be added to the database of tire tread prints and used for matching with tire tread prints which are found at crime scene.

The classification of tire tread images by using intuitionistic fuzzy set functions was made in some papers also before. For example, in [6] authors used similarity measure defined on intuitionistic fuzzy sets for classification of the images. For similarity measures there could be found many counter-intuitive results, see [8]. In the mentioned paper, the authors suggest new 




a)

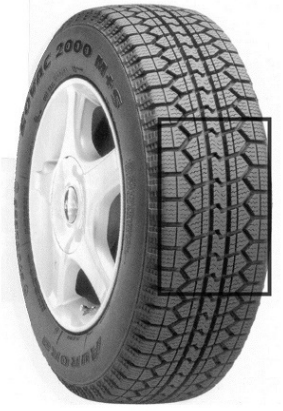

b)



c)

Figure 2. Visualization of position of tire tread print

function, so called Sim function, to measure similarity of two intuitionistic fuzzy sets. This function is defined as a ratio between distance of two intuitionistic fuzzy sets and distance between the complement of the first intuitionistic fuzzy set and the second intuitionistic fuzzy set. Similarly, in [7], the authors calculated Sim function as the ratio of distance between pattern data and image data and distance between pattern data and the complement of image data. The complement of image data was obtained by using specific intuitionistic fuzzy negation. In [2] a list of 53 intuitionistic fuzzy negations was presented. We have decided to use some of these negations to improve the results of classification.

The paper is structured as follows. In Section 2 we give a brief introduction into the theory of intuitionistic fuzzy sets. In Section 3 we discuss the way how we could prepare the data for classification. In Section 4 the obtained results are summarized and in Section 5 the conclusions are mentioned.

\section{Intuitionistic fuzzy sets}

Intuitionistic fuzzy sets (shortly IFSs) were introduced by Krassimir Atanassov in 1983 [1]. Since then, many new properties and applications of this mathematical structure have been constructed. In this section we define the functions and properties of intuitionistic fuzzy sets which are used in this paper.

Definition 1. Let $X$ be the universe. An intuitionistic fuzzy set is a set

$$
A=\left\{\left\langle x, \mu_{A}(x), \nu_{A}(x)\right\rangle \mid x \in X\right\}
$$

of the functions $\mu_{A}: X \rightarrow[0,1], \nu_{A}: X \rightarrow[0,1]$ such that $0 \leq \mu_{A}(x)+\nu_{A}(x) \leq 1$.

Function $\mu_{A}$ is called the membership function and function $\nu_{A}$ is called the non-membership function.

Denote by $\mathcal{F}$ the family of all IFSs. Then there exists another one function defined on $\mathcal{F}$, function $\pi_{A}$ which is defined as

$$
\pi_{A}(x)=1-\mu_{A}(x)-\nu_{A}(x) .
$$

This function is called hesitation margin. 
Considering the character of the function $\pi_{A}$, an intuitionistic fuzzy set $A$ could be represented as a couple $\left(\mu_{A}(x), \nu_{A}(x)\right)$ and also as a triple $\left(\mu_{A}(x), \nu_{A}(x), \pi_{A}(x)\right)$. In this paper both notations are used.

Definition 2. Let us have two intuitionistic fuzzy sets $A=\left(\mu_{A}, \nu_{A}\right), B=\left(\mu_{B}, \nu_{B}\right)$. Then it holds

$$
\begin{aligned}
& A=B \Longleftrightarrow \mu_{A}=\mu_{B} \& \nu_{A}=\nu_{B} \\
& A \leq B \Longleftrightarrow \mu_{A} \leq \mu_{B} \& \nu_{A} \geq \nu_{B}
\end{aligned}
$$

One of the ways how to classify the images is to use the similarity measure, which for IFSs is defined as follows:

Definition 3. Let $S$ be a real-valued function such that $S: \mathcal{F} \times \mathcal{F} \rightarrow[0,1]$. $S$ is called a similarity measure if for every $A, B, C \in \mathcal{F}$ it holds

- $S(A, B)=S(B, A)$.

- $S(A, B)=1$ iff $A=B$.

- If $A \leq B \leq C$ then $S(A, C) \leq S(A, B)$ and $S(A, C) \leq S(B, C)$.

There exist various kinds of functions which fulfil the properties of similarity measures. Each of them could give different result of classification. In paper [8], the authors specified examples of counter-intuitive results for various types of similarity measures. In the same paper, the authors suggested another approach to compare the IFSs. They computed the distance between two IFSs $A$ and $B$ and distance between $A$ and $B^{C}$, where $B^{C}$ is a complement of $B$ and they asked the question:

\section{Is A more similar or more dissimilar to B?}

This approach is used also in this paper. To calculate the distance between two elements (pattern and image) the distance function defined on IFSs is used.

Definition 4. Let $d$ be a real-value function such that $d: \mathcal{F} \times \mathcal{F} \rightarrow[0, \infty]$. Function $d$ is called distance function defined on IFSs if for every $A, B, C \in \mathcal{F}$ it holds

- $d(A, B)=d(B, A)$.

- $d(A, B)=0$ iff $A=B$.

- If $A \leq B \leq C$ then $d(A, C) \geq d(A, B)$ and $d(A, C) \geq d(B, C)$.

As a tool to measure if some element $A$ (which represent the pattern) is more similar or more dissimilar to $B$ (which represent the image) authors in paper [8] defined function Sim:

Definition 5. Let $A, B, B^{C} \in \mathcal{F}$, where $A=\left(\mu_{A}(x), \nu_{A}(x), \pi_{A}(x)\right), B=\left(\mu_{B}(x), \nu_{B}(x), \pi_{B}(x)\right)$ and $B^{C}$ is a complement of $B$. Let $d$ be the distance function defined on $\mathcal{F}$, then

$$
\operatorname{Sim}(A, B)=\frac{d(A, B)}{d\left(A, B^{C}\right)} .
$$


Theorem 6. For function Sim it holds

- $0 \leq \operatorname{Sim}(A, B) \leq \infty$,

- $\operatorname{Sim}(A, B)=0$ means identity of $A$ and $B$,

- $\operatorname{Sim}(A, B)=1$ means $A$ is to same extent similar to $B$ and $B^{C}$,

- $\operatorname{Sim}(A, B)>1$ means $A$ is more similar to $B^{C}$ than to $B$,

- $d\left(A, B^{C}\right)=0$ means complete dissimilarity of $A$ and $B$.

Proof. See [8].

To analyze the similarity of two IFSs $A$ and $B$, the values $\operatorname{Sim}(A, B) \in[0,1)$ are interesting.

Also various kinds of distance functions are defined on IFSs. In this paper we have used normalized Euclidean distance function

$$
d_{E}(A, B)=\left(\frac{1}{2 n} \sum_{i=1}^{n}\left(\left(\mu_{A}\left(x_{i}\right)-\mu_{B}\left(x_{i}\right)\right)^{2}+\left(\nu_{A}\left(x_{i}\right)-\nu_{B}\left(x_{i}\right)\right)^{2}+\left(\pi_{A}\left(x_{i}\right)-\pi_{B}\left(x_{i}\right)\right)^{2}\right)\right)^{\frac{1}{2}} .
$$

In the paper [7] as the complement to element $B=\left(\mu_{B}(x), \nu_{B}(x), \pi_{B}(x)\right)$ the triple $B^{C}=\left(\nu_{B}(x), \mu_{B}(x), \pi_{B}(x)\right)$ was used.

Let $A=\left(\mu_{A}, \nu_{A}\right)$ be an intuitionistic fuzzy set. Then its complement could be obtained by using arbitrary intuitionistic fuzzy negation. The most commonly used intuitionistic fuzzy negation is negation referred as standard intuitionistic fuzzy negation, $\neg_{1}$ and for it $\neg_{1}(A)=\left(\nu_{A}(x), \mu_{A}(x)\right)$ holds. This negation was used also in [7]. In [2], 53 kinds of different intuitionistic fuzzy negations were defined. These negations could be divided into some specific groups:

1. Negations which used just one coordinate of IFS $A$, for example

$\neg_{4}(A)=\left(\nu_{A}(x), 1-\nu_{A}(x)\right)$ or

$\neg_{8}(A)=\left(1-\mu_{A}(x), \mu_{A}(x)\right)$.

2. Negations which used value of function signum of one coordinate of IFS $A$, for example

$\neg_{2}(A)=\left(1-\operatorname{sgn}\left(\mu_{A}(x)\right), \operatorname{sgn}\left(\mu_{A}(x)\right)\right)$ or

$\neg_{5}(A)=\left(1-\operatorname{sgn}\left(1-\nu_{A}(x)\right), \operatorname{sgn}\left(1-\nu_{A}(x)\right)\right)$, where

$\operatorname{sgn}(x)=\left\{\begin{array}{l}1, \text { if } x>0 \\ 0, \text { if } x \leq 0\end{array}\right.$

3. Negations which used both coordinates of IFS $A$, for example $\neg_{3}(A)=\left(\nu_{A}(x), \mu_{A}(x) \cdot \mu_{A}(x)+\mu_{A}(x) \cdot \nu_{A}(x)\right)$.

4. Negations which used value of function signum of both coordinates of IFS $A$, for example $\neg_{6}(A)=\left(1-\operatorname{sgn}\left(1-\nu_{A}(x)\right), \operatorname{sgn}\left(\mu_{A}(x)\right)\right)$.

5. Negations which used coordinates of IFS $A$ and some real valued parameter, for example $\neg 42, \lambda(A)=\left(\frac{\nu_{A}(x)+\lambda-1}{2 \lambda}, \frac{\mu_{A}(x)+\lambda}{2 \lambda}\right)$, where $\lambda \geq 1$. 
The main idea for preparing of this paper was that different types of intuitionistic fuzzy negations could cause different quality of classification. For example, if someone used that kind of negation which consist just one coordinate from the original IFS $A$, then some information is lost. On the other hand, if the intuitionistic fuzzy negation consist function signum, then the obtained values for $A^{C}$ are strictly 0 or 1 . In the presented research we used first four kinds of negations. We did not used negations with parameters. Notes, that some negations without parameters are specific kind of those negations with parameters. For example if we use $\lambda=1$ in negation $\neg_{42, \lambda}$ then we got the formula

$$
\neg_{42,1}(A)=\left(\frac{\nu_{A}(x)}{2}, \frac{\mu_{A}(x)+1}{2}\right)=\neg_{35}(A) .
$$

From this point of view also the negations with specific parameter were used.

\section{Preparation of the data}

As it was mentioned in the introduction, we downloaded the images of tire treads from different web pages. We need to prepare them in such way that we are able to process them by using intuitionistic fuzzy sets distance function. The process of adaptation of data into the right format is called pre-processing and in this research it could be divided into two parts. The first part is pre-processing of the images, the second part is pre-processing of the data.

\subsection{Pre-processing of the images}

For pre-processing of the images we developed the algorithm which consists of the following four steps

1. Converting image into the JPEG format.

2. Removing white pixels on all sides of the image.

3. Converting image into the black and white format.

4. Dividing image into 16 parts.

In the fourth step the image of tire tread is divided into 16 rectangles as it could be seen on Figure 3 . Then the image is represented by 16 coordinate vector where each coordinate represents the number of white pixels in given part.

\subsection{Pre-processing of the data}

Now the second part of adaptation - pre-processing of the data - could start. To calculate the value of membership and non-membership function to each coordinate of the image vector, we used the procedure that was described in the paper [4]. To build the database of templates and patterns, we used the same approach as it was mentioned in papers [6] and [7]. For each of the seven classes mentioned in Introduction we choose the so called templates. Template images 


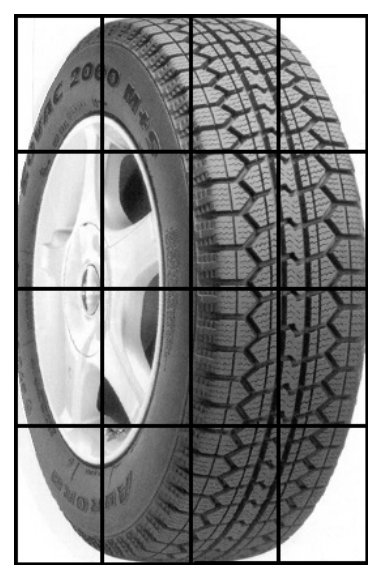

Figure 3. Dividing the image into 16 parts

represented their classes. From each class 3 images as the templates were selected. Therefore, there were used 21 templates which were represented by 16 coordinate vectors.

Let us have template $i(i=1,2, \ldots, 21)$ represented by a 16 -coordinate vector

$$
T_{i}=\left(x_{i, 1}, x_{i, 2}, \ldots, x_{i, 16}\right)
$$

We started with the normalization of each coordinate by using formula

$$
n_{i, j}=\frac{x_{i, j}-\bar{X}_{j}}{s_{j}}
$$

where $j=1,2, \ldots, 16, \bar{X}_{j}$ is the mean and $s_{j}$ is the standard deviation calculated from the $j$-th coordinate of all images in the template database. Then the membership degree of each template coordinate is calculated by the weighted sigmoid function

$$
\mu_{i, j}=\frac{r_{j}}{1+e^{-n_{i, j}}}
$$

where $r_{j}$ is a weight value. Similarly, the non-membership degree of each template coordinate is calculated by the formula

$$
\nu_{i, j}=\frac{r_{j}^{*}}{1+e^{n_{i, j}}} .
$$

In the end the value of hesitance margin of each template coordinate is calculated by the formula

$$
\pi_{i, j}=1-\mu_{i, j}-\nu_{i, j}
$$

In [8], the authors used different combination of values of the weights $r_{j}$ and $r_{j}^{*}$ from unit interval (specifically combination of the values $0 ; 0.1 ; 0.2 ; \ldots ; 1$ ). The most satisfying results were obtained for values $r_{j}=1$ and $r_{j}^{*}=0.6$. We also used these values of the weights $r_{j}$ and $r_{j}^{*}$.

Since we were dividing the images into the seven classes we defined the seven patterns by the formula

$$
P_{m}=\left\{\left\langle\bar{x}_{m, j}, \bar{\mu}_{m, j}, \bar{\nu}_{m, j}, \bar{\pi}_{m, j}\right\rangle\right\}_{j=1}^{16},
$$

where $m=1,2, \ldots, 7$ and the values $\bar{\mu}_{m, j}, \bar{\nu}_{m, j}$ and $\bar{\pi}_{m, j}$ represent the arithmetical mean of function values of the templates which belong to given class. 


\subsection{Classification of the images}

After pre-processing of the data we were ready to classify any image. We used following algorithm

1. Take any image.

2. Use pre-processing and characterize image by 16-coordinate vector (see Section 3.1).

3. For each coordinate calculate the value of membership function, non-membership function and hesitation margin by the same formulas as were used for templates (see Section 3.2).

4. Calculate the distance between the image and each of seven patterns.

5. Classify image into the suitable class by using the Sim function.

The image was classified into that class where the value of Sim function between the image and pattern was the lowest.

\section{Experimental results and discussion}

For the experiment we took 350 images which were downloaded from the web pages with the different names of tire brand. We developed a software that pre-processed the images by using the above mentioned algorithms. As a result, the program creates seven folders (each folder represents one class) and moves the images into the folders as they were classified by the described process. To help us quickly identify the incorrectly classified images, there was also given one template image into the each created folder. Program creates also the text file in which the information about the value of Sim function was written.

When the standard negation $\neg_{1}$ for compute image data complement was used, then the classification reached the value $78.6 \%$ of correctness. The worst results were obtained by using negations $\neg_{37}$ (reached the value $64.0 \%$ of correctness) and $\neg_{36}$ (reached the value $64.3 \%$ of correctness). These negations had the common property, that they contain the membership and non-membership value of the IFS $A$ divided by value 3 , specifically

$$
\neg_{37}(A)=\left(\frac{2 \nu_{A}(x)}{3}, \frac{2 \mu_{A}(x)+1}{3}\right) \text { and } \neg_{36}(A)=\left(\frac{\nu_{A}(x)}{3}, \frac{2+\mu_{A}(x)}{3}\right) .
$$

The second worst results were obtained by using negations $\neg_{38}$ (reached the value $66.0 \%$ of correctness) and $\neg_{39}$ (reached the value $69.7 \%$ of correctness). These negations had again the common property, that they contain one of the values - the membership or non-membership value of the IFS $A$ divided by value 3 , specifically

$$
\neg_{38}(A)=\left(\frac{1-\mu_{A}(x)}{3}, \frac{2+\mu_{A}(x)}{3}\right) \quad \text { and } \neg_{39}(A)=\left(\frac{\nu_{A}(x)}{3}, \frac{3-\nu_{A}(x)}{3}\right) .
$$


On the other hand, the best results were obtained by using negation $\neg_{3}$ (reached the value $84.6 \%$ of correctness). This negation contains the combination of the membership and non-membership value of the IFS $A$, specifically

$$
\neg_{3}(A)=\left(\nu_{A}(x), \mu_{A}(x) \cdot \mu_{A}(x)+\mu_{A}(x) \cdot \nu_{A}(x)\right) .
$$

The values of correctness of classification are presented in the Table 1 . There are missing the values of the correctness of the negations $\neg_{42}-\neg_{51}$. These negations depend on one or more parameters and therefore it was not possible to indicate the correctness of classification for these negations.

\begin{tabular}{|c|c|}
\hline Correctness of classification & Used negations \\
\hline $84.6 \%$ & $\neg 3$ \\
\hline $84.3 \%$ & $\neg_{52}$ \\
\hline $83.7 \%$ & $\neg_{7}, \neg_{9}$ \\
\hline $83.4 \%$ & $\neg_{15}, \neg_{16}, \neg_{17}$ \\
\hline $83.1 \%$ & $\neg 33$ \\
\hline $82.9 \%$ & $\neg_{28}$ \\
\hline $82.6 \%$ & $\neg_{8}, \neg_{11}, \neg_{13}, \neg_{14}, \neg_{21}, \neg_{22}, \neg_{23}, \neg_{31}, \neg_{32}$ \\
\hline $82.3 \%$ & $\neg_{19}, \neg_{20}, \neg_{25}, \neg_{27}, \neg_{29}, \neg_{30}, \neg_{35}$ \\
\hline $80.9 \%$ & $\neg_{26}$ \\
\hline $80.3 \%$ & $\neg_{10}$ \\
\hline $80.0 \%$ & $\neg_{12}$ \\
\hline $78.6 \%$ & $\neg_{1}, \neg_{18}, \neg_{53}$ \\
\hline $77.7 \%$ & $\neg_{4}, \neg_{24}, \neg_{34}$ \\
\hline $75.4 \%$ & $\neg 40$ \\
\hline $73.1 \%$ & $\neg_{2}, \neg_{5}, \neg_{6}$ \\
\hline $71.4 \%$ & $\neg_{41}$ \\
\hline $69.7 \%$ & $\neg_{39}$ \\
\hline $66.0 \%$ & $\neg_{38}$ \\
\hline $64.3 \%$ & $\neg_{36}$ \\
\hline $64.0 \%$ & $\neg 37$ \\
\hline
\end{tabular}

Table 1. Result of classification

As it was mentioned before, during the running of application, the value of Sim function was written into a text file. We could compare obtained results also considering these values. We could conclude that the greater the value of correctness was, the smaller the values of Sim function for the individual images were. For the negations with the value of correctness greater than $80.0 \%$ the values of Sim function for individual images reached values from interval $[0.1,0.3]$. In this group of classification, the value of $\mathrm{Sim}$ function did not reach a value greater than or equal 1 for any image. For the negations with the small value of correctness, the values of Sim function for individual images reach mostly the values from interval $[0.4,0.7]$. In this group of classification, 
the value of Sim function reached for some images also value greater than 1 . The images with values of Sim function greater than 1 were classified incorrectly according to the property of Sim function, that if the value of Sim function is greater than 1, then the complement of this image is closer to pattern as the original image. This could help us identify the images which are incorrectly classified or images which represent such objects that could be hardly classified into the one group.

Similarly, as in [6] and [7] there were some incorrectly classified images which had some common properties. There were some images in the database, for which the human expert also finds it hard to classify them exactly into one class. For example, the image on Figure 4a was frequently classified into Class 1 but image on Figure $4 \mathrm{~b}$ was frequently classified into the Class 4 . The next example is the image on the Figure $4 \mathrm{c}$ which was classified into the Class 7 . Another problem is if there is too much captured light on the image. For example, image on Figure 4d was classified into the Class 1 but it actually belongs to Class 5 . The worst results reach the classification of the text (Class 6). Into this class images with much captured light were also assigned, for example the image on Figure 4e.

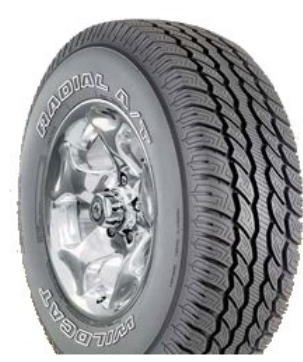

(a)

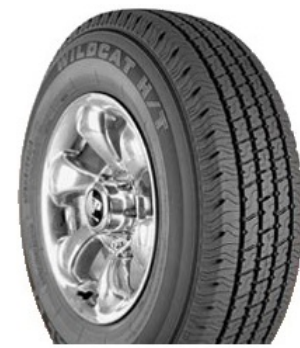

(b)

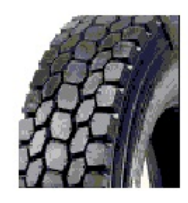

(c)

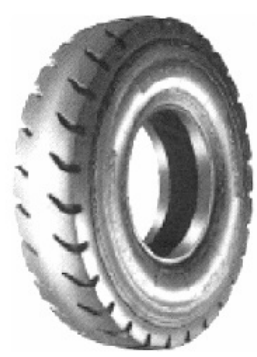

(d)



(e)

Figure 4. Images mentioned in discussion

\section{Conclusions}

In this paper, we used intuitionistic fuzzy sets for the classification of tires tread images. We described the way how the image could be represented by the vectors. Then for each vector we determined the value of membership and also the value of non-membership function. We used the Euclidean distance function to calculate the distance between the image with the predetermined patterns. We classified the set of images by using Sim function and different kinds of intuitionistic fuzzy negations and discussed the results and problems of incorrect classification. Moreover, another advantage of this approach is, that it could be used in automated processing of the images which are obtaining from the web pages.

\section{Acknowledgements}

The support of the grant KEGA 006UMB-4/2020 is kindly announced. 


\section{References}

[1] Atanassov, K. T. (1983). Intuitionistic Fuzzy Sets, VII ITKR Session, Sofia, 20-23 June 1983 (Deposed in Centr. Sci.-Techn. Library of the Bulg. Acad. of Sci., 1697/84) (in Bulgarian). Reprinted: Int. J. Bioautomation, 2016, 20 (S1), S1-S6.

[2] Atanassov, K. T. (2017). Intuitionistic Fuzzy Logics, Springer International Publishing AG 2017, Switzerland. DOI 10.1007/978-3-319-48953-7.

[3] Bodziak, W. J. (2017). Forensic Footwear Evidence, CRC Press.

[4] Intarapaiboon, P. (2016). Text classification using similarity measures on intuitionistic fuzzy sets. SCIENCEASIA, 42 (1), 52-60.

[5] Lux, F. H. (2013). Tire Track Identification, Journal of Forensic Research, Vol. 4:198. doi:10.4172/2157-7145.1000198.

[6] Michalíková, A. (2019). Intuitionistic Fuzzy Sets and Their Use in Image Classification, Notes on Intuitionistic Fuzzy Sets, 25 (2), 60-66.

[7] Michalíková, A. (2019). Classification of images by using distance functions defined on intuitionistic fuzzy Sets, in Advances in Intelligence Systems and Computing. Warsaw, Poland. Springer. Submitted.

[8] Szmidt, E., \& Kacprzyk, J. (2009). Analysis of Similarity Measures for Atanassov's Intuitionistic Fuzzy Sets, in IFSA/EUSFLAT Conference, pp. 1416-1421.

[9] Vagač, M., Melicherčík, M., Marko, M., Trhan, P., Michalíková, A., Kliment R. \& Drapka, R. (2015). Crawling Images with Web Browser Support. 13th International IEEE Scientific Conference on Informatics'2015, 286-289.

[10] Vagač, M., Melicherčík, M. \& Schon, J. (2015). Classification of Tire Images in Order to Obtain the Best Possible Tire Tread Sample. The 5th International Scientific Conference, Applied Natural Science 2015. September 30-October 2, 2015, Jasná; Trnava : UCM, 173. 